\title{
RADIO FREQUENCY HEATING COILS FOR SHOCK WAVE EXPERIMENTS
}

\author{
GEORGE Q. CHEN* AND THOMAS J. AHRENS**
}

*Present address: The Santa Cruz Operations, Inc., 400 Encinal Street, POB 1900, Santa Cruz, CA 95061-1900

**Correspondent: Lindhurst Laboratory of Experimental Geophysics, Seismological Laboratory 252-21, California Institute of Technology, Pasadena, CA 91125

\section{ABSTRACT}

Radio-frequency eddy current heating of metallic samples in shock wave, ultrasonic and diamond anvil apparatus provides a methodology for obtaining hot samples or hot metallic gaskets (containing a sample) and yet not heating the entire target, ultrasonic assembly or diamond cell. Analysis of a previous design of a radio-frequency $(\sim 0.5 \mathrm{MHz})$ coil demonstrated that the center of $13 \mathrm{~mm}$ diameter shock wave experiment sample discs were underheated and the experimental temperatures achieved resulted from conduction of heat from the overheated sample edges relative to the center of the sample. We show, using the Biot-Savant law, that the ratio of magnetic field in the center to that at the edge of the sample, $f$, can be maximized to a value of $f=1.8$, by decreasing the radius of the heater coil, relative to the $13 \mathrm{~mm}$ diameter of sample. A simple analysis provides the thermal gradient across the sample thickness. An electric skin depth for iron of $\sim 1 \mathrm{~mm}$ for the $0.45 \mathrm{MHz}$ frequency of the heater power supply of our system is obtained. This leads to a maximum temperature gradient of $\sim 52 \mathrm{~K}$ over the $4 \mathrm{~mm}$ thickness of the shock wave sample discs.

\section{INTRODUCTION}

The heating coil is an important part in preheated shock-wave experiments and the ideas presented here apply as well to ultrasonic and diamond anvil methodology. Heating uniformity at high temperature was more critical to the Chen and Ahrens [1] study of the preheated iron equation of state and high pressure and high temperature sound velocity measurement experiments than previous studies [2]. This is because it was necessary to heat solid targets to as close to the melting point as possible in order to lower the shock pressure required to achieve melting.

\section{Maxwell Equations}

We employ the Maxwell equations to develop a simple model for optimizing the radiofrequency induction in the target.

$$
\begin{gathered}
\operatorname{div} \mathbf{D}=\rho, \\
\operatorname{div} \mathbf{B}=0, \\
\operatorname{curl} \mathbf{E}=-\frac{\partial \mathbf{B}}{\partial t}, \\
\operatorname{curl} \mathbf{H}=\mathbf{J}+\frac{\partial \mathbf{D}}{\partial t},
\end{gathered}
$$

where $\mathbf{B}$ and $\mathbf{E}$ are electric and magnetic fields, $\mathbf{H}=\mathbf{B} / \mu$ is the magnetic intensity, and $\mu$ is the magnetic permeability; $\mathbf{D}=\boldsymbol{\varepsilon E}$ is the electric displacement, $\varepsilon$ is the electric permittivity, $\rho$ is electric charge density, and $\mathbf{J}$ is electric current density. For vacuum, $\varepsilon_{0}=8.85 \times 10^{-12} \mathrm{farad} / \mathrm{m}$ and $\mu_{0}=$ $4 \pi \times 10^{-7}$ henry $/ \mathrm{m}$.

Equation 3 dictates that inside any medium, when there is an alternating magnetic field, an electric field is induced. If the medium is a conductor, an eddy current, of density: 


$$
\mathbf{J}=\beta \mathbf{E},
$$

where $\beta$ is the conductivity, will be present. This current, in turn, heats up the conductor. The heat per unit volume generated by the current is:

$$
\mathrm{q}=\mathrm{J}^{2} / \beta
$$

This is the basis of induction heating of metals. For a sinusoidally oscillating $B$ field, $q \propto$ $\mathbf{J}^{2} \propto \mathbf{E} \propto \mathbf{B}$.

In our pre-heated experiments, the (external) alternating magnetic field is generated by the heating coil according to the Biot-Savart law (see, e.g., Slater and Frank [3]):

$$
\mathbf{B}=\frac{\mu_{0}}{4 \pi} \frac{\mathrm{I} \mathbf{d s} \times \mathbf{r}}{\mathbf{r}^{3}}
$$

where $\mathbf{r}$ is the location of interest from a length $\mathbf{d s}$ of wire carrying a current $\mathrm{I}$.

This field is coupled to the inside of the metal as required by Equations 2 and 4 . For the $B$ component normal to the metal surface, Equation 2 dictates that $B_{\perp}$ is continuous across the metal surface; for the parallel component $B_{1}$, Equations 4 requires $H_{4}=B_{l} / \mu$ is continuous (for air, $\mu \cong$ $\left.\mu_{0}\right)$. Therefore, by evaluating the external magnetic field distribution at the metal surface, we can estimate the temperature field in the target. This is demonstrated in the following section.

However, we do not make an attempt to estimate the coupling efficiency of the coil and the target as this is a complex problem, and the coupling attained in our experiments is quite adequate.

\section{Coil Design}

It was found that the original coil design [2] (using $6 \mathrm{~mm}$ (1/4 inch) outer-diameter copper tubing, coil, inner diameter, $\sim 50 \mathrm{~mm}$ ) was not suitable for high temperature iron experiments in which the sample is $13 \mathrm{~mm}$ in diameter and $4 \mathrm{~mm}$ thick [1].

To improve the heating of the target, the coil was redesigned. Unfortunately, since the geometry of the coil is complicated, only a detailed numerical solution can predict the electromagnetic field on the target surface, the generation of heat, its conduction and radiation away from the heated zone. However, we used a few simple formulae from electromagnetic theory to understand how the coil geometry affects target heating.

1. The magnetic field on the axis of the coil, approximated by a single circular current flow (Figure 2), and is given by the Biot-Savart law [3]:

$$
\mathrm{B}_{\text {center }}=\frac{\mu_{0} \mathrm{Ia}^{2}}{2\left(\mathrm{a}^{2}+\mathrm{x}^{2}\right)^{3 / 2}}
$$

Equation 8 applies to a constant current flow, or an alternating current of amplitude I, in which case $B_{\text {center }}$ is also the amplitude of the alternating field. The latter interpretation is applicable to our problem. 


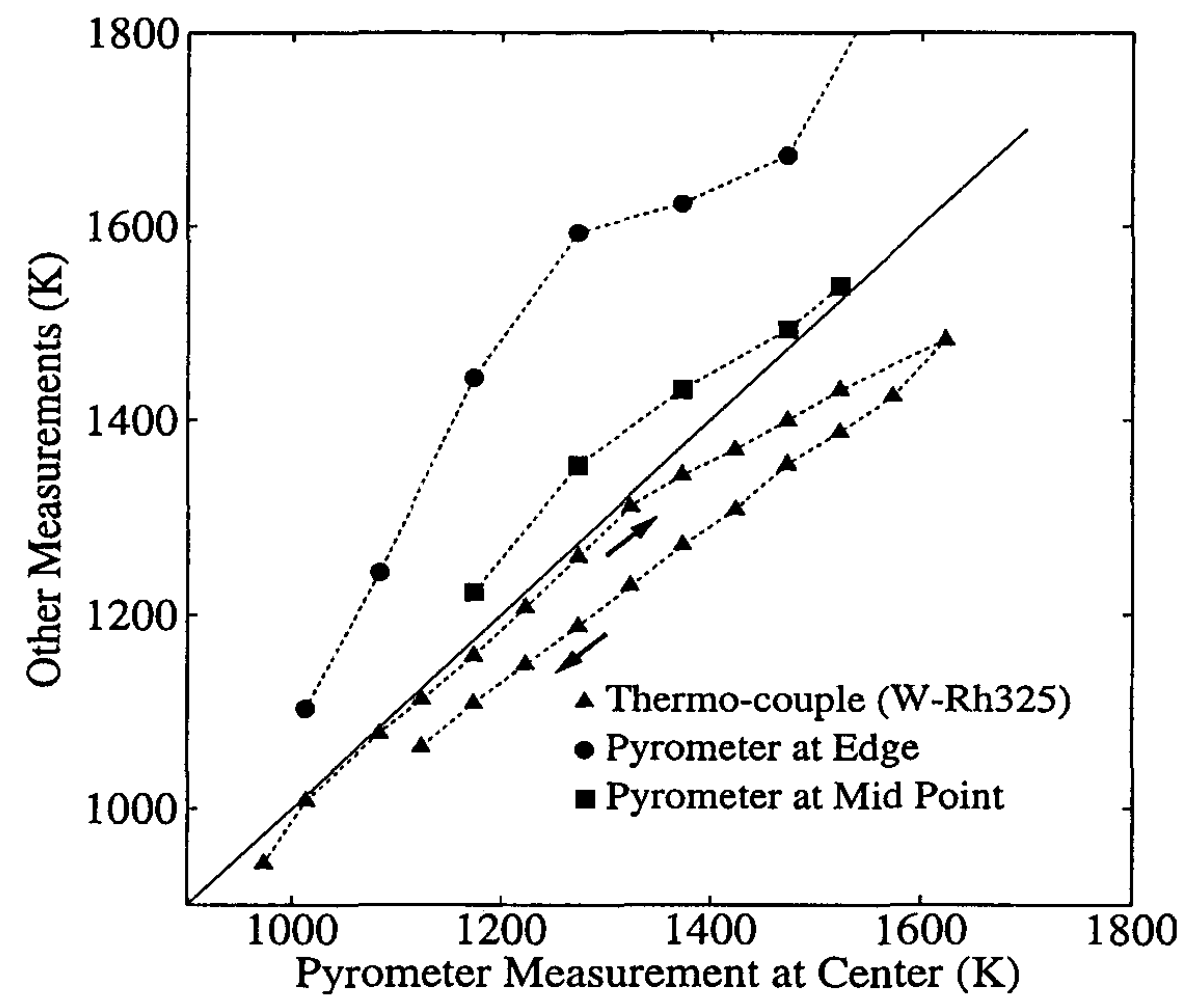

Figure 1: Temperature distribution in an iron target heated with $50 \mathrm{~mm}-\mathrm{I}$.D. coil. Temperature measured at target center using a Williamson 8120 S-C-WD2 pyrometer $\left(45^{\circ}\right.$ line) is shown relative to temperatures measured by other means. A radial temperature gradient is demonstrated by the deviation of edge and mid-point temperatures on driver plate from central temperature. The target edge temperature approaches the melting point of iron at $1811 \mathrm{~K}$, when central temperature reaches $\sim 1500 \mathrm{~K}$. Measurements made with thermocouple are also shown. Arrows indicate heating history. Thermocouple well is on driver plate, about $1 \mathrm{~mm}$ from top hat (Figure 2).

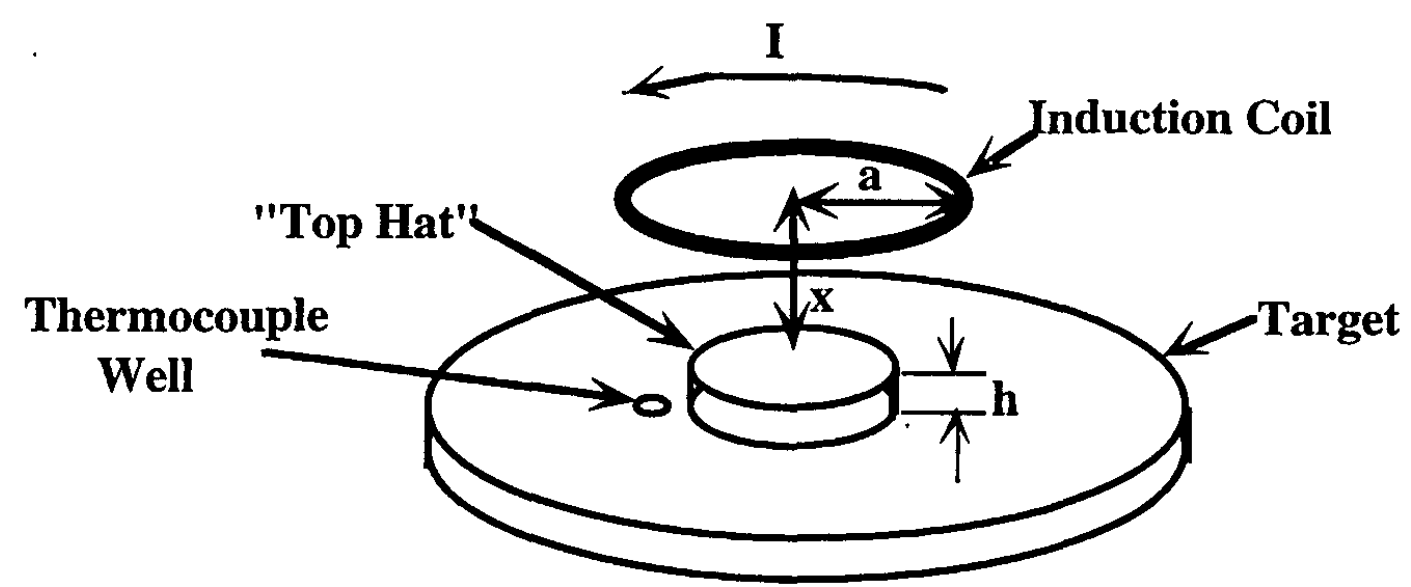

Figure 2: Simplification of equating the linear induction current described by Equation 8 (Fig. 3) to a circular current flow $\mathrm{I}$. Here $\mathrm{h} \sim 4 \mathrm{~mm}$ and $\mathrm{t} \sim 2 \mathrm{~mm}$ for present targets. a and $\mathrm{x}$ are to be determined. Thermocouple well is a $\sim 1 \mathrm{~mm}$ diameter hole drilled $\sim 1 \mathrm{~mm}$ deep. 
2. For estimating $B$ off the center line, we approximate the magnetic field induced by the coil to that was produced by an infinite straight line current (Figure 3). Since the coil is close to the target surface (coil-target distance $(\mathrm{h}+\mathrm{x})<2 \mathrm{a}$ ), we expect this approximation to be valid [3]. From Equation 7 we obtain:

$$
B_{\text {edge }}=\frac{\mu_{0} I}{2 \pi(x+h)}
$$

$\mathrm{B}_{\text {edge }}$ is defined as the amplitude of the alternating field.

We note from Fig. 1 that the temperatures induced in our targets are such that these are greatest at the target edge and least in the target center. We therefore require a coil that will provide a stronger magnetic field at the center of the sample.

Thus, we need to calculate a few cases using Equations 8 and 9 in order to understand how to increase the factor, $\mathrm{f}$ :

$$
\mathrm{f}=\mathrm{B}_{\text {center }} / \mathrm{B}_{\text {edge }} \text {. }
$$

1. We first calculate the fields for the previous coil design. Although $f>1$ at $x>4.2 \mathrm{~mm}$ (Figure 4), the experiments show the center of the target is under-powered. This is probably because the multiple coil windings (used to boost heating at any given power setting) induced a magnetic field strength $B_{\text {edge }}$ that exceeds $B_{\text {center }}$. Notice $f$ does not reach its maximum even when $x=10 \mathrm{~mm}$, at such large values of $x$, the coupling between the coil and the target is unacceptably low. We need to find a solution to increase $f$.

2. The obvious solution is to decrease both $\mathrm{a}$ and $\mathrm{x}$. A calculation for $\mathrm{a}=12.5 \mathrm{~mm}$ is shown in Figure 4. Here $f$ reaches its maximum at $x=6 \mathrm{~mm}$.

The new coil design (Figure 5) necessitates the use of $3 \mathrm{~mm}(1 / 8 \mathrm{inch})$ outer diameter tubing. We used a template with two Plexiglas discs and a center rod (Figure 6) to control the coil inner diameter, and $3 \mathrm{M}$ heat shrink tubing to wrap tightly around the copper tubing to control the winding spacing. With the new coil, the maximum temperature difference over the entire target is less than $40 \mathrm{~K}$ at $1573 \mathrm{~K}$ (Figure 7). The new design also demonstrates improved power coupling between the target and the coil.

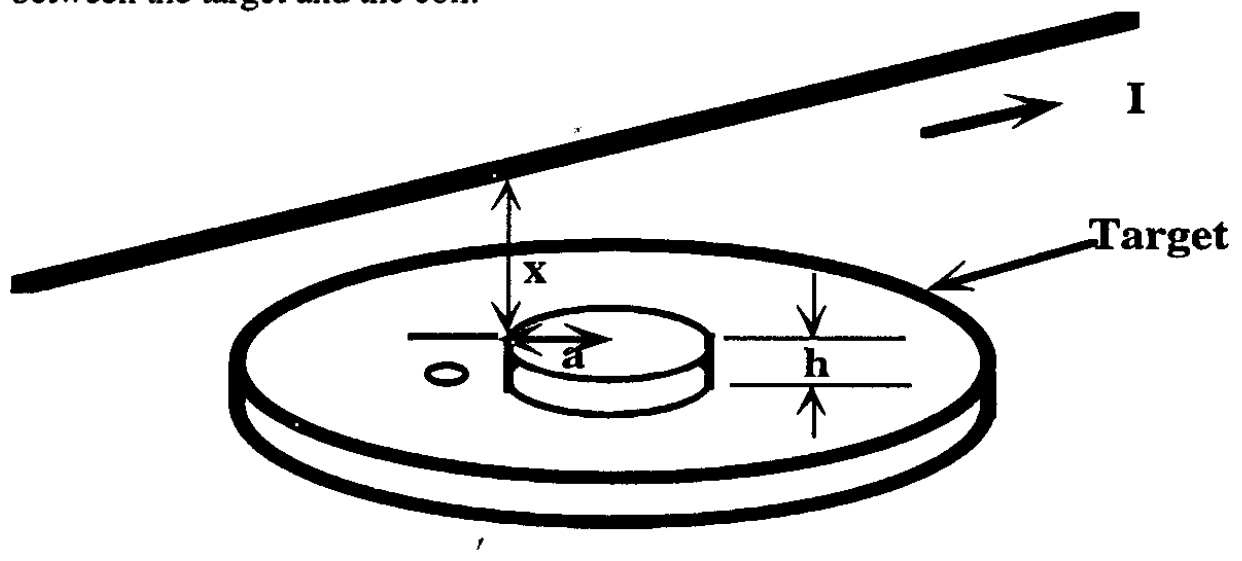

Figure 3: Approximation of effect of coil at off-center positions to that of an infinite straight line current I parallel to driver plate. Dimensions a and $\mathrm{x}$ are to be determined. 

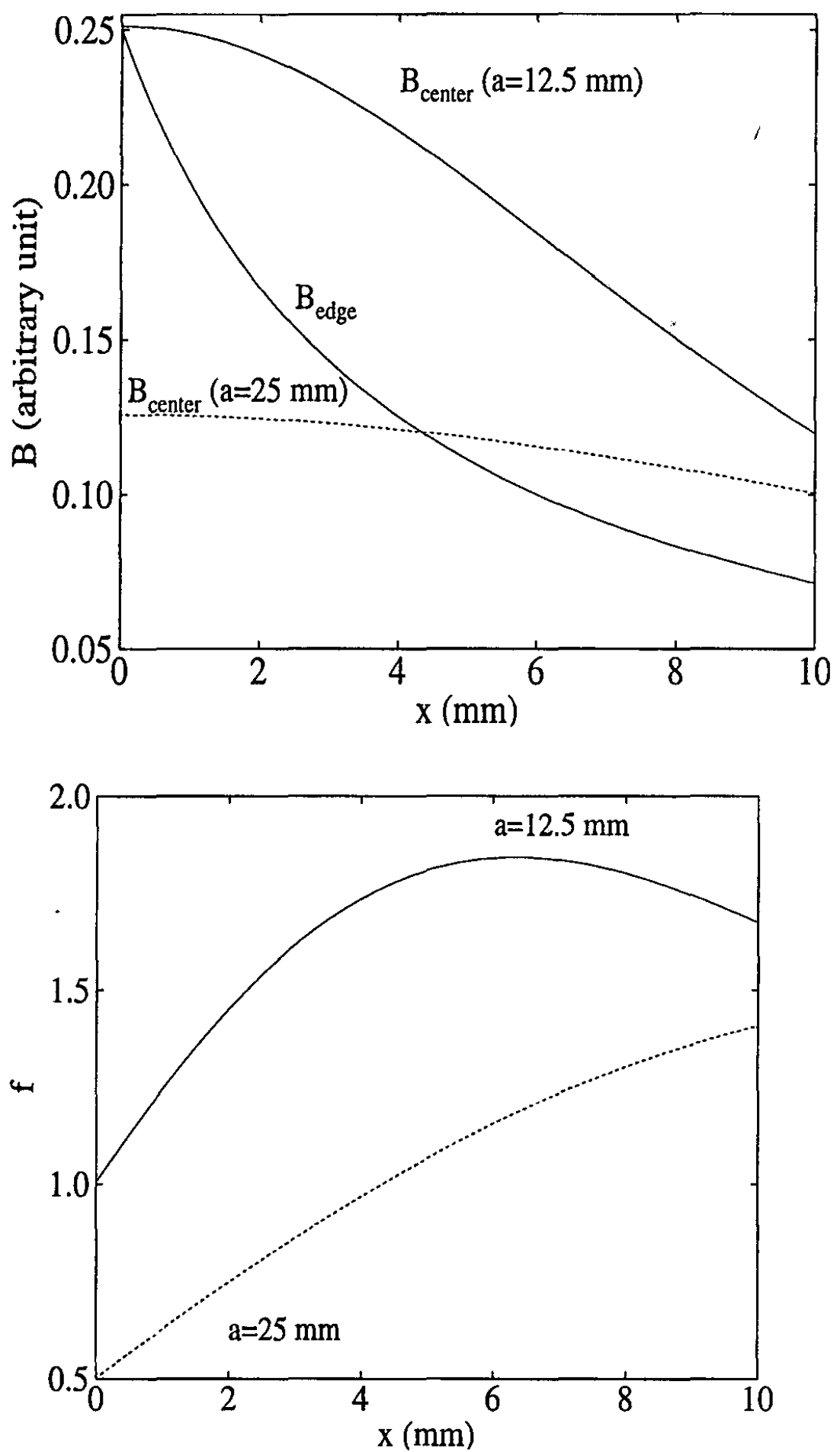

Figure 4: Calculations of magnetic fields and factor $\mathrm{f}$ for $\mathrm{a}=25 \mathrm{~mm}$ (previous coil design) and $\mathrm{a}=$ $12.5 \mathrm{~mm}$ (new coil design). Edge field strengths in two cases are identical, but the smaller coil significantly boosts central field strength. 


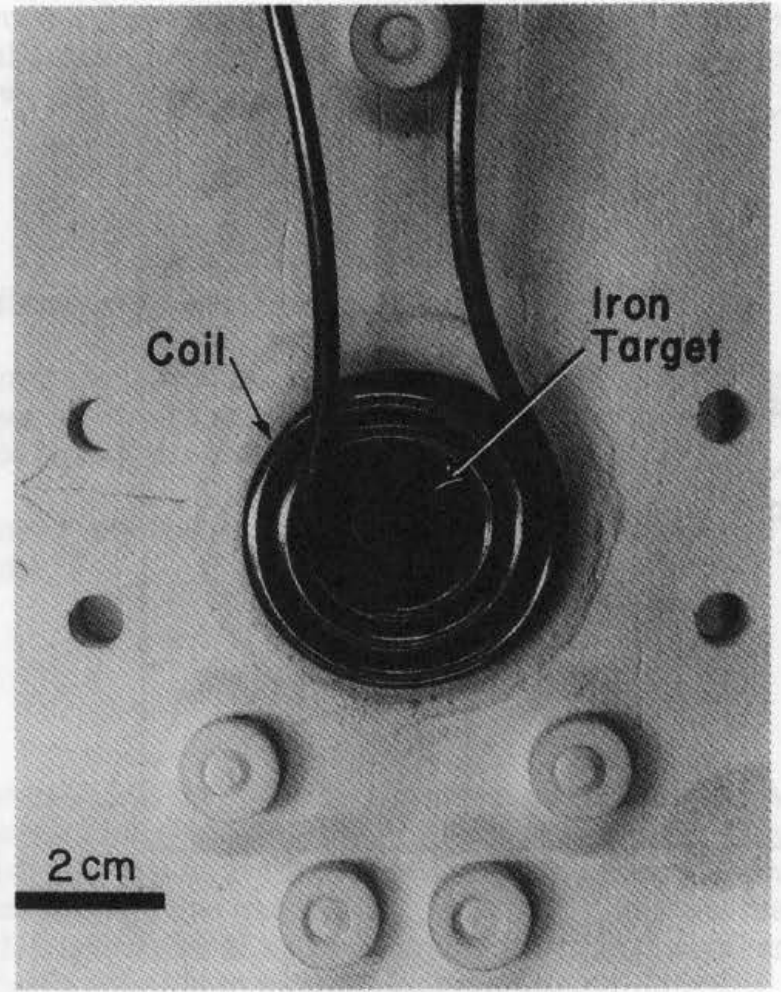

Figure 5: Heating coil used in preheated iron experiments.

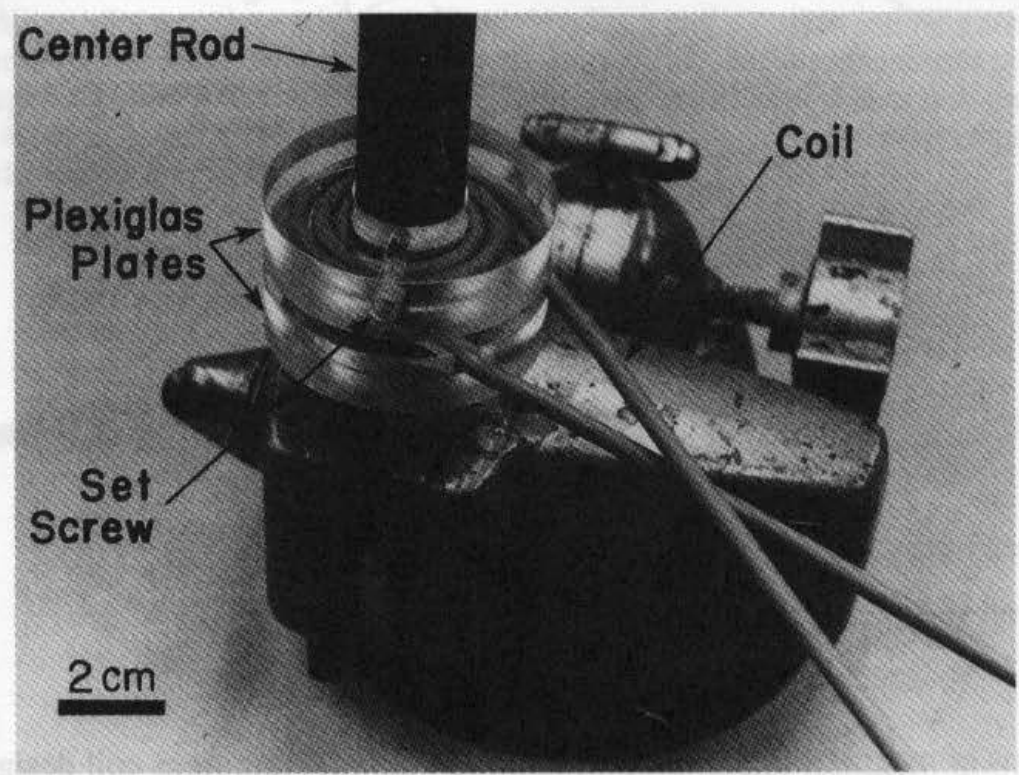

Figure 6: Photo of template used to make heating coil. 


\section{Temperature Heterogeneity}

The temperature gradient across the lateral surface of the target is measured experimentally as described in Sect. 2. However, there is also a gradient along the thickness of the target, due to the limited skin depth of metal that the electromagnetic field can penetrate [3]:

$$
\delta=\sqrt{\frac{2}{\omega \beta \mu}}
$$

where $\omega$ is the circular frequency of the oscillating field.

For an order-of-magnitude estimate, $\beta=1.8 \times 10^{6} \Omega^{-1} \mathrm{~m}^{-1}$ for iron at $800 \mathrm{~K}$ (values at higher temperatures are not available) [4]. Assuming $\mu=\mu_{0}$, the penetration depth is $1.0 \mathrm{~mm}$ at the $450 \mathrm{kHz}$ frequency of the RF heater. Thus, $\delta$ is less than the target thickness used $(\mathrm{h}+\mathrm{t} \approx 6 \mathrm{~mm})$.

A simple model is used to estimate the effect of varying the penetration depth. On the unheated side of the target, the rate of energy loss by thermal radiation per unit area of target surface is given by:

$$
\mathrm{E}_{1}=\sigma \mathrm{T}^{4}
$$

where $\sigma=5.671 \times 10^{-8} \mathrm{~W} \mathrm{~m}^{-2} \mathrm{~K}^{-4}$ is the Stefan-Boltzmann constant. Assuming a temperature difference $\Delta \mathrm{T}$ exists in the layer of iron between the penetration depth and the unheated surface (of thickness $h+t-\delta$ ), the rate of heat flow per unit area across a control surface at the RF penetration depth, $\delta$, is:

$$
E_{2}=\frac{\gamma \Delta T}{h+t-\delta}
$$

where $\gamma$ is iron's thermal conductivity. At equilibrium, $E_{1}=E_{2}$ yields

$$
\Delta \mathrm{T}=\frac{\sigma \mathrm{T}^{4}(\mathrm{~h}+\mathrm{t}-\delta)}{\gamma}
$$

For Fe, $\gamma=32.8 \mathrm{~W} \mathrm{~m}^{-1} \mathrm{~K}^{-1}$ at $1573 \mathrm{~K}$ [4], we calculate that $\Delta \mathrm{T}=52 \mathrm{~K}$. For the Mo capsules used in the liquid silicate experiments [2], the temperature difference should be less $(\approx 20 \mathrm{~K})$ because convection within the liquid greatly reduces the temperature difference.

\section{CONCLUSIONS}

A $10 \mathrm{~kW}$ R-F $(450 \mathrm{kHz})$ induction heater drives a $12.8 \mathrm{~mm}$ diameter coil placed $12.5 \mathrm{~mm}$ above a metallic target (e.g. iron). The coil has one complete turn plus a partial turn, yields a radial temperature uniformity of $\pm 20 \mathrm{~K}$ at $1573 \mathrm{~K}$. The temperature variation through a $7 \mathrm{~mm}$ thick iron target assembly is calculated to $\sim 50 \mathrm{C}$ at the centerline. Previous $25 \mathrm{~mm}$ diameter coils overheated the edge of $30 \mathrm{~mm}$ diameter target plates. 
We note that analyses of the present work, although motivated by the needs of the shock wave experimentor, can also be applied to radio-frequency heating of ultrasonic and diamond anvil sample assemblies.

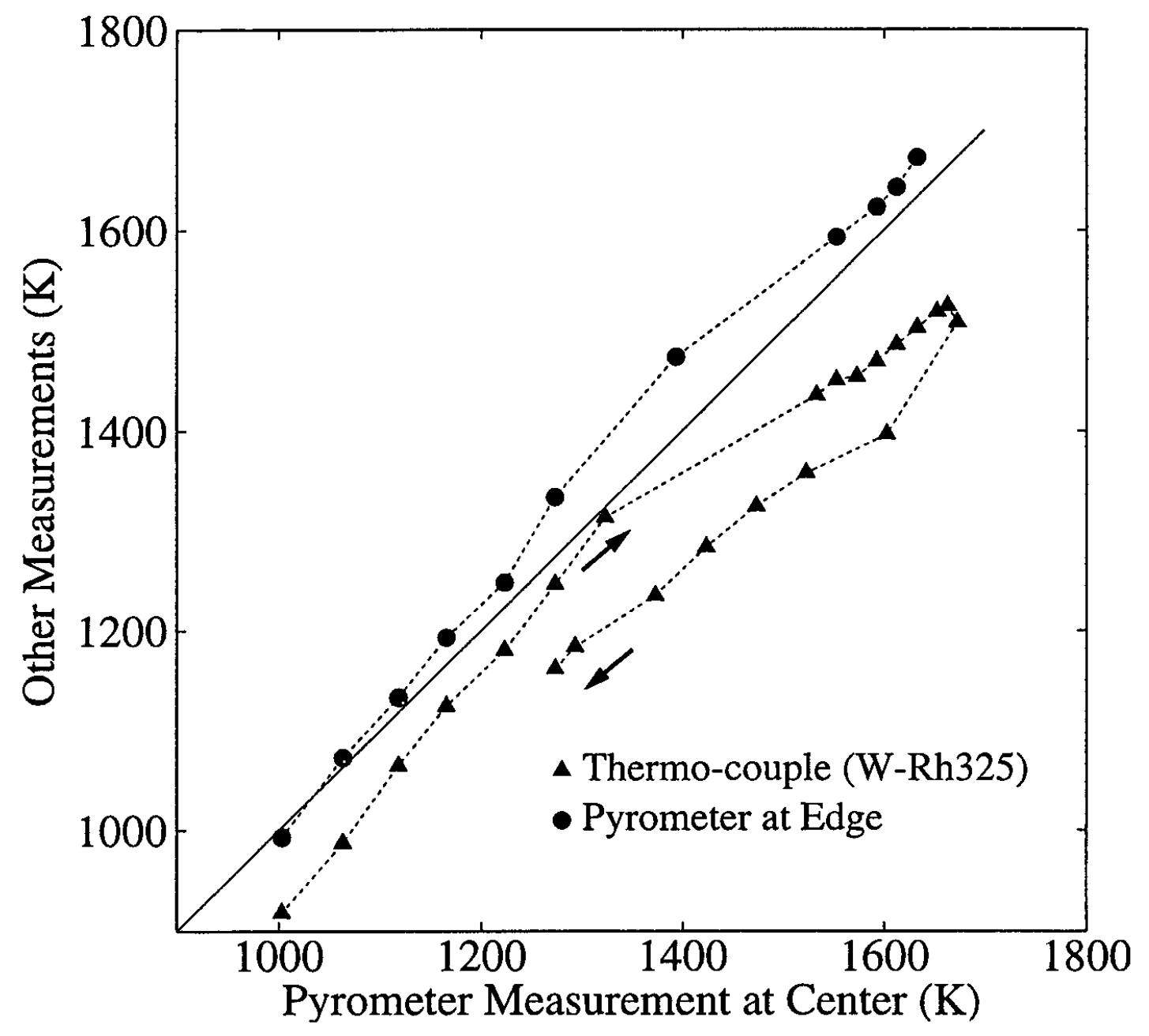

Figure 7: Temperature distribution in an iron target heated with a $12.5 \mathrm{~mm}$-radius coil. Temperature measured at target center using pyrometer as a reference for temperatures. Arrows indicate heating history.

\section{ACKNOWLEDGMENTS}

Research supported by NSF. We thank E. Gelle, M.Long, and A. Devora for experimental assistance. Contribution \#8499, Division of Geological and Planetary Sciences, California Institute of Technology, Pasadena, California.

\section{REFERENCES}

[1] G.Q. Chen and T.J. Ahrens, High pressure and high temperature equation-of-state of gamma and liquid iron, in These Proceedings, p._to_, Materials Research Society Pittsburgh 1998. 
[2] S.M. Rigden, T.J. Ahrens and E.M. Stolper, Shock compression of molten silicates: Results for a model basaltic composition, J. Geophys. Res., 93, 367-382, 1988.

[3] J.C. Slater and N.H. Frank, Electromagnetism, Dover Publications, Inc., New York, NY, 1969.

[4] D.R. Lide, Handbook of Chemistry and Physics, Vol. 74th Edition, edited by 4-70, CRC Press, Boca Raton, Florida, 1993-1994. 